\title{
Complete Heart Block and Pregnancy Outcome: An Analysis from Eastern India
}

\author{
Saroj Mandal, Debasmita Mandal*, Achyut Sarkar, Jagat Biswas and Manotosh Panja \\ Department of Obstetrics \& Gynecology, Institute of Cardiovascular Sciences (ICVS), IPGME\&R, SSKM Hospital, 244 AJC \\ Bose Road, Kolkata, 700020, West Bengal, India
}

Received: May 13, 2015; Accepted: June 29, 2015; Published: July 15, 2015

*Corresponding author: Dr. Debasmita Mandal, QR.NO.C/11, SSKM Hospital Campus, 242, A.J.C. Bose Road, Kolkata, 700020, West Bengal, India, Tel: 9831522415, 03322232615; E-mail: drdebasmitamondal@yahoo.com, debasmita_mandal@rediffmail.com

\begin{abstract}
Objective: Complete heart block is rarely experienced with pregnancy. We assessed indications requiring pacemaker, types and route of pacing and reviewed foeto-maternal outcome with obstetric management.

Methods: This is a longitudinal prospective cohort study from a tertiary referral institute in Eastern India. Pregnant women with CCHB ( $\mathrm{n}=21$ women with 28 pregnancies) were included as subjects. All patients underwent relevant investigations and vigilant antenatal checkups. Pacing is carried out through right internal jugular vein approach after scrutinizing the actual need.

Results: Syncope was observed in 6 (29\%) women (second trimester and during or after delivery). Echocardiography revealed abnormalities like: single ventricle in one and Congenitally Corrected Transposition of Great arteries in two cases. Seventy six percent women delivered with pacemaker. Thirty-six percent required temporary pacemaker (TPM) in intrapartum period, $11 \%$ had permanent pacemaker (PPM) prior pregnancy and another $11 \%$ had PPM implantation during second trimester of pregnancy. All of the 5 postnatal cases were instituted PPM subsequently. Mean gestational age at delivery was $38 \pm 3$ weeks. Eighty six percent pregnancies were delivered by vaginal route. Except few adverse outcome pregnancy outcome was favorable. None had neonatal CHB.

Conclusion: Pregnancy with CHB can be complicated with syncope during any phase of pregnancy. Permanent pace maker implantation is safe and better option in early pregnancy to avoid life threatening complications later. Cesarean section should be only reserved for obstetric indications. Multidisciplinary approach with tertiary care back up can achieve successful pregnancy outcome in patients with CHB.
\end{abstract}

Keywords: CHB; Pregnancy outcome; Maternal bradycardia; Temporary pacemaker; Permanent pacemaker

\section{Introduction}

Complete Heart Block (CHB) was reported in 1901, by Morquio who described a familial occurrence [1,2]. It is a conduction disorder and characterized by a random relationship between atrial and ventricular activation where atrial impulses are not conducted to ventricle. Incidence of CHB either complete or with more than $50 \%$ of blocked atrial impulses, has been estimated at 1 in 15,000 to 20,000 live birth [2,3]. It can be completely idiopathic and can also be a result of passive autoimmunity with cardiac ailment in response to active transport of maternal IgG autoantibodies to fetal circulation, as in SLE. The history of CHB begins in utero and there is a tendency for female preponderance [2-4]. One study group followed up 102 subjects with CHB during adulthood and observed in lifelong perspective and explained it to be a life threatening disease with significant morbidity and risk of Stokes Adams (SA) attacks and sudden death at any age could be anticipated even in the absence of poor prognostic signs [5]. Patients with isolated CHB, who survive infancy are usually asymptomatic and achieve normal growth and development although mortality in this period is $8 \%$ [2]. Thus cases associated with pregnancy may be observed. CHB is rare and relatively serious problem in pregnancy as feto-maternal outcome is concerned. A normal physiologic response to pregnancy creates an increased cardiac output by 30 to $50 \%$ by increased heart rate and blood volume. These alterations pose a substantial challenge in the setting of fixed maternal heart rate. As pregnancy and CHB is a rare association and reported in the literature as small series or isolated case reports, there are no established guidelines regarding management.

Here we are sharing our experience of 21 women with CHB during pregnancy and analyzed the pregnancy outcome in terms foeto-maternal morbidity and mortality and discussed the management issues.

\section{Materials and Methods}

This was a prospective cohort study of consecutive CHB patients hospitalized to the Department of Cardiology and Obstetrics and Gynecology of IPGME\&R, SSKM Medical College Hospital, between March 2002 and February 2013. It's a 1600 bed largest tertiary care centre and university hospital in Eastern India. The cardiology and obstetric services receive approximately 50000 and 15000 patients annually for cardiological and antenatal care respectively. Here a total of 21 women aged between 19 and 39 years with 28 pregnancies (15 first pregnancies and 13 second pregnancies) were analyzed. All patients were diagnosed to have CHB with singleton pregnancies were included in the study. Since we want to concentrate only 
on association of CHB with pregnancy, we excluded all other risk factors (multiple pregnancy, CHB patients presenting with any other medical disorder) that can influence the outcome. This inclusion had two categories of patients; CHB patients registered in the department of cardiology and conceived subsequently and pregnant women presenting with symptoms and signs of CHB in emergency outpatient department and diagnosed thereafter. CHB was diagnosed according to clinical symptoms and Electrocardiography.

We elicited a detailed history from the patients and referring doctors by relevant questionnaires. All had routine laboratory investigations like; total hemogram, Liver Function Test (LFT), renal function test, blood sugar profile and coagulation profile. Serial ultrasonography was performed as routine procedure to monitor fetal growth and other maternal parameters that can suggest the pregnancy outcome. Collagen profile was routinely done to rule out connective tissue disorder as a cause of CHB. Maternal echocardiography was also carried out as routine procedure to exclude any associated congenital heart disease or left ventricular dysfunction as a sequel of long standing CHB. Fetal echocardiography was done at 22 to 24 weeks to diagnose any congenital affection. Women were admitted if they develop any pregnancy and disease related complications or when they were on their expected date of delivery. Close surveillance of labor was done with continuous monitoring of maternal heart rate and periodic blood pressure measurement. Pacing was carried out after proper clinical and investigational evaluation of the patients according to guidelines by AHA [6]. They were followed up vigilantly to determine the need of pacemaker. As few of our patients had already on implanted pace makers, temporary pacemaker support was given depending on the history of syncope, near syncope or fatigability. These cases were analyzed with respect to the time of presentation, feto maternal morbidities and treatment given during peripartum period. Obstetric events were defined by standards listed in William Obstetrics, $22^{\text {nd }}$ edition [7].

Informed consent was obtained from all participants of the study. This study was ethically cleared from our institutional ethical committee. The study is adhered to declaration of Helsinki.

\section{Results}

Twenty one patients with complete arterio ventricular block (prenatally diagnosed and already had Permanent Pacemaker or patients who presented with complete AV block antenatlly, during labor and in puerperium) were included in the study. Only 5 women with CHB were registered in Department of Cardiology with regular follow up.

Table 1 summarizes the epidemiology and parity of the patients along with onset and route of delivery. Twenty-one patients had 28 pregnancies, of them 14 women were nulliparous and seven were multiparous. Most of the patients were in age group 20 to 30 years and none of them was above 40 years. Sixteen of our subjects presented antenatally and majority of the pregnancies delivered vaginally.
Table 2 shows clinical presentation at the time of admission. Syncopal attack (Stokes-Adam-attack) was observed in four women during delivery and each of the two during second trimester and postpartum period. Most of the patients were in NYHA class I symptom throughout the course of the pregnancy, except four who had NYHA class II and III status. Although all patients were a cyanotic, easy fatigability was a common symptom. Minimum pulse rate was 30 in the study population and in majority heart rate ranged between 40 and 50 (Table 3). Jugular venous pressure showed canon waves in 16 patients. Table 4 depicts status of investigations at presentation. Regarding QRS duration, only one patient had wide QRS complex and a heart rate at $30 / \mathrm{min}$ and all others were observed to have narrow QRS complex.

All cases had normal collagen profile. Echocardiography revealed structurally normal heart with situs solitus, normal atrioventricular and ventriculoarterial concordance. Among them three patient had moderate left ventricular dysfunction (LVEF: $35-40 \%$ ) and another two showed Congenitally Corrected Transposition of Great arteries (CCTGA) while single ventricle was observed in one patient.

Table 5 reveals details of pacemaker implantation. Sixteen

Table 1: Demography characteristics of women with CHB.

\begin{tabular}{|c|c|c|}
\hline Factors & $\begin{array}{c}\text { No of patients } \\
(\mathbf{n = 2 1 )}\end{array}$ & Percentage (\%) \\
\hline Age & & \\
\hline $19-29$ & 8 & 38 \\
\hline $30-39$ & 13 & 62 \\
\hline$>40$ & None & $-\cdots-$ \\
\hline Parity & & 67 \\
\hline P1+0 & 14 & 29 \\
\hline P2+0 & 6 & 5 \\
\hline P3+0 & 1 & 86 \\
\hline Route of delivery & No of preg (n=28) & 14 \\
\hline VD* & 24 & \\
\hline LSCS* & 4 & 57 \\
\hline Time & 16 & 18 \\
\hline Antenatal & 5 & \\
\hline Postnatal & \multicolumn{2}{|c|}{} \\
\hline VD: Vaginal Delivery*, LSCS: Lower Segment Cesarean Section* \\
\hline \multicolumn{2}{|r|}{} \\
\hline
\end{tabular}

\begin{tabular}{|c|c|c|}
\hline \multicolumn{3}{|c|}{ Table 2: Clinical presentation of pregnant women with CHB. } \\
\hline $\begin{array}{c}\text { Clinical } \\
\text { features }\end{array}$ & No of patients (n= 21) & Percentage (\%) \\
\hline Syncope & 6 & 29 \\
\hline Palpitation & 8 & 38 \\
\hline Fatigability & 21 & 100 \\
\hline Dyspnea & 4 & 19 \\
\hline NYHA III & 4 & 19 \\
\hline
\end{tabular}


mothers delivered under pacemaker coverage [10- Temporary Pace Maker (TPM) during intrapartum period, 3- permanent pacemaker (PPM) during second trimester and 3- PPM prior to pregnancy]. Rest five patients had PPM after delivery. In the CCTGA patient, delivery was carried out under TPM support which was done one day prior to induction of labor and the procedure was instituted though Right internal jugular approach and temporary lead placed in right sided ventricle which is morphologically left ventricle.

Table 6 depicts feto-maternal outcome the study population. Median gestational age at delivery was 38 weeks among live births (ranged between 28 and 40 weeks). Median birth weight was 3020gm (ranged between 1550and 3992gm). All except few delivered vaginally. Cesarean section was reserved for obstetrics indications only. The length of the hospital stay was approximately 3 to 5 days. Adverse outcome included pre-term labor (11\%), syncopal related injury (4\%) and oligohydramnios (7\%). Among 28 live births no baby had congenital affection.

\section{Discussion}

Congenital heart block is a rare reason for fetal bradycardia [8]. Though prevalence of CHB is relatively more in females, symptomatic CHB in young pregnant females is an unusual association [9]. Thirty percent of the CHB cases remain

Table 3: Clinical examination findings of pregnant patients with CHB.

\begin{tabular}{|c|c|c|}
\hline Findings & No of patients $(\mathbf{n = 2 1 )}$ & Percentage (\%) \\
\hline Cyanosis & none & --- \\
\hline Pulse/min & & \\
\hline $30-40$ & 4 & 19 \\
$40-50$ & 14 & 67 \\
$50-60$ & 3 & 14 \\
\hline JVP canon & 16 & 76 \\
wave & & \\
\hline
\end{tabular}

Table 4: Investigations at admission of pregnant women with CHB.

\begin{tabular}{|c|c|c|}
\hline Investigations & No of patients $(n=21)$ & Percentage (\%) \\
\hline \multicolumn{3}{|l|}{ ECG } \\
\hline Complete AV dissociation & 21 & 100 \\
\hline $\begin{array}{c}\text { Narrow QRS }(60-80 \mathrm{~mm}) \\
\text { Wide } \mathrm{ORS}(>80 \mathrm{~mm})\end{array}$ & 20 & $\begin{array}{c}95 \\
5\end{array}$ \\
\hline \multicolumn{3}{|l|}{ Echocardiography } \\
\hline Situs solitus & 21 & 100 \\
\hline LAE & 4 & 19 \\
\hline LVE & 4 & 19 \\
\hline LV Dysfunction & 3 & 14 \\
\hline AV/VA concordance & 18 & 86 \\
\hline AV/VA Discordance & 2 & 10 \\
\hline Single ventricle & 1 & 5 \\
\hline CCTGA* $^{*}$ & 2 & 10 \\
\hline \multicolumn{3}{|c|}{${ }^{*}$ Congenitally Corrected Transposition of Great arteries } \\
\hline
\end{tabular}

Table 5: Details of pacemaker implantation during delivery of women with CHB.

\begin{tabular}{|c|c|c|}
\hline Delivery with pacemaker & $\begin{array}{c}\text { No of patients ( } \\
\mathbf{n = 1 6 )}\end{array}$ & Percentage (\%) \\
\hline With TPM* support & 10 & 63 \\
\hline PPM* prior to pregnancy $^{\text {PPM during pregnancy }}$ & 3 & 19 \\
\hline $\begin{array}{c}\text { Post delivery PPM } \\
\text { implantation }\end{array}$ & 3 & 19 \\
\hline Within 1 month & 3 & 60 \\
\hline After 1 month & 2 & 40 \\
\hline $\begin{array}{c}\text { *TPM: Temporary Pace Maker } \\
\text { *PPM: Permanent Pace Maker }\end{array}$ & & \\
\hline
\end{tabular}

\begin{tabular}{|c|c|c|}
\hline Table 6: Feto-maternal outcome in maternal CHB. \\
\hline Maternal outcome & $\begin{array}{c}\text { No of pregnancies } \\
\text { (n=28) }\end{array}$ & $\begin{array}{c}\text { Percentage } \\
\mathbf{( 1 0 0 \% )}\end{array}$ \\
\hline Pre term labor & 3 & 11 \\
\hline Syncope related injury & 1 & 4 \\
\hline Oligohydramnios & 2 & 7 \\
\hline APH* & 1 & 4 \\
\hline Fetal outcome & 25 & 89 \\
\hline Term delivery & 3 & 11 \\
\hline Prematurity & 4 & 14 \\
\hline FGR* & 0 & 18 \\
\hline Fetal heart block & 5 & \\
\hline NICU admission & & FGR: Fetal Growth Restriction \\
\hline *APH: Ante Partum Hemorrhage, &
\end{tabular}

asymptomatic until adulthood and may present first time during pregnancy and puerperium [9]. The study has been analyzed in terms of demography of study group, profile of CHB patients, their pregnancy outcome and issues related to pacemaker application.

In the present series during nine years of study, we followed up 21 mothers with 28 pregnancies. Congenital complete heart block accounted for 2.4 per 1000 deliveries. All patients had block at atrioventricular node. Conception was spontaneous in all. All patients had complete AV dissociation and narrow QRS in ECG. Strokes Adams attack (SA attack) or syncope was observed in $28.57 \%$ of cases mainly during second stage of labor and postpartum. Literature related SA attacks with MR, long QTc in CHB patients. Low VRs, widened QRS, low exercise response and ectopies were not predictive of SA [5]. During pregnancy SA attacks might be related to a blunted response of a diseased heart to the maternal physiological alteration which is yet to explore. Generally cardiac output in pregnancy is increased by 30 to $50 \%$ as a result of raised heart rate, stoke volume, blood volume and decrease in the peripheral resistance. But in situation like CHB; the slow heart rate, long standing filling period and increased end diastolic volume and fiber length augment myocardial contractile force and thereby results in increased stoke volume, which maintains the cardiac output. So pregnancy 
is well tolerated certain times without syncope. But chronically high stroke volume in the non gravid state, results in dilated ventricle and which creates a limited capacity to further augment end-diastolic volume and stroke volume. This hemodynamic alteration is unable to acclimatize with the increase cardiac output in pregnancy $[10,11]$.

That's why pacing in CHB with pregnancy whether comes under the necessary step of management or not is a debatable issue. Hidaka $\mathrm{N}$ et al. concluded that women with AV block and without permanent pacing do not routinely require temporary pacing for labor and birth. They had inserted the pacemaker lead but used it only when patient become symptomatic [12]. Some other authors suggested that symptomatic patients should undergo PPM in first trimester only or before conception [13-15]. But in the patients near or at term, TPM would be a preferred method of pacing just before the induction of labor or during labor [9]. Kharde VK et al. had put forth some criteria for temporary pacing ie; atropine resistant bradycardia, first and second degree AV block and atrial fibrillation with low ventricular rate [10]. Modi et al. suggested that though asymptomatic patients with exercise tolerance can be managed without pacemaker in pregnancy, but there should be an available arrangement of TPM [16]. Vanita Suri et al. had an experience of four pregnancies with CHB and observed two patients requiring TPM and rest two who delivered without support of pacemaker had syncope within four months of delivery [17]. Michaělesson $M$ et al. studied 102 adult cases, among them 24 women were pregnant who delivered 45 children. They concluded that as SA attack was a life threatening risk in CCHB patients, so early PPM implantation not only proved life saving but also might reduce morbidity like mitral valve insufficiency [5].

ACC/AHA guidelines indicated pacemaker implantation in cases with third degree AV block in adults with syncope, second and advanced third degree AV block with associated symptomatic bradycardia, congestive heart failure and decreased cardiac output [6]. In the present series all patients were evaluated and followed up individually to determine need for TPM / PPM. Sixteen antenatals delivered under pacemaker coverage. Indications of pacing included ie; maternal bradycardia non responsive to medication, patients with left ventricular dysfunction and presence of history of syncope in preconception, antenatal and postnatal period. Eleven patients had PPM implantation, five in the postpartum phase and another three in early and late second trimester. Three women had PPM before pregnancy. Ten pregnancies required temporary pacemaker coverage. Temporary pacing was carried out through Right jugular vein approach. Considering the patient position during delivery transfemoral venous approach was avoided. Women who could not afford dual chamber pacing, single chamber pacing was done and before delivery routine programming of the pacemaker was carried out and heart rate was fixed at higher side. Second trimester PPM was done with minimal fluoroscopic and radiation exposure.

Though pacing has got important role, small limitations cannot be ignored. They are, safety of pacemaker implantation in first trimester and fixed rate of pacing, which may be detrimental if demand during pregnancy increases more than it. However above literatures have suggested that pacing is safe with minimum fluoroscopic and radiation exposure. This has been taken care of nowadays, by improved implantation techniques, better achievable cardiovascular function than conventional medical therapy. In our series most of the PPM are from 17-18 Rate Responsible pacemakers (VVIR: ventricular pacing, ventricular screening, inhibition, rate responsive) (3D pacemakers). A simple VVIR pacing system in early pregnancy will obviate the need for temporary pacing by the femoral route later in pregnancy. Limitation of this study is small sample. We could not conclude any significant pregnancy outcome differences between the women who had pacemaker prior pregnancy and pacemaker during pregnancy except Stoke Adam's attack which was more observed in later group.

After pacing next steps of management in pregnancy with CHB include route of delivery and anesthesia and analgesia. In the present study $86 \%$ had vaginal delivery and $14 \%$ underwent cesarean section for obstetric indications. Our CCTGA patient delivered vaginally at 35 weeks with a healthy baby. Foetomaternal outcome included syncope (29\%), pre-term labor $(11 \%)$, fetal growth restriction (14\%), oligohydramnios $(7 \%)$ and postpartum hemorrhage ( $4 \%$ ). We feel that fetal growth restriction arises due to chronic maternal bradycardia leading to fetal hypoxia. Cases with heart ranged between 30 and 40 and cases with Stoke Adams attack were observed to suffer from FGR and oligohydramnios. No neonatal CHB was observed. Few other authors had also similar observation $[9,12,17]$. Contrary to these outcome in patients with CHB due to autoimmunity (anti-Ro/SSA antibodies) have worse prognosis [18].

In this series second stage was curtailed by instrument application when required. All of our patients with operative interferences had epidural anesthesia and analgesia. Modi et al. depicted epidural to be the safe mode of anesthesia provided they had continuous ECG monitoring with TPM in hand [16]. Another observer suggested that sudden onset of high sympathetic blockade with spinal anesthesia results in bradycardia and reflex cardiovascular depression with vasodilatation due to disease itself causing more severe hypotension. So instrumental epidural tops with low dose combined spinal epidural anesthesia can be recommended during operative interferences [17].

Because of the retrospective analysis of few women who presented postnatally, we could not conclude any firm opinion regarding relationship of foeto-maternal outcome with CHB. Quantitative measurement of foetal hypoxia if any could not be assessed due to unavailability of resources.

All pregnant patients with CHB should undergo regular and vigilant antenatal checkups. Any antenatal patient who presents fatigability and syncope should be stress for an ECG which is a noninvasive and cost effective way to diagnose this disease. Though pregnancy is tolerable with CHB, circumstances like Stokes Adams attack cannot be overlooked which actually necessitates delivery under pacemaker coverage. Any female with 
CHB presenting in early pregnancy or planning for conception, PPM implantation irrespective of symptoms or other markers of risk (syncope, hemodynamic compromise at third trimester, at delivery and in postpartum period) is desirable. To make it mandatory needs further studies with large sample. Favorable pregnancy outcome can be expected with due time pacemaker implantation and multidisciplinary tertiary care approach from cardiologist, obstetrician and neonatologist.

\section{Acknowledgement}

We acknowledge Prof. Dr. Abhijit Hazra for a real contribution to our study as a biostatician.

\section{References}

1. Morquio L. Sur une maladieinfantile et familialcaractérisée par des modeficationspermanents du pouls, des aattaquessyncopales et epileptiform et al mortsubite. Arch Méd d’Enfants. 1901; 4: 467.

2. Perloff JK. The clinical Recognition of Congenital Heart Disease, 5thed. Philadephia: Elsevier; 2003.

3. Macado MVL, Tyanan MJ, Curry PVL, Allan LD. Fetal complete heart block. Br Heart J. 1988; 60: 512.

4. Esscher EB. Congeital complete heart block in adolescence and adult life: A follow-up study. Eur Heart J. 1981; 2(4): 281-8.

5. Michaëlsson M, Jonzon A, Riesenfeld T. Isolated Congenital Complete Arterioventricular Block in Adultlife: A Prospective study. Circulation. 1995; 92(3): 442-3

6. Gregoratos G, Cheitlin MD, Conill A, Epstein AE, Fellows C, Ferguson TB $\mathrm{Jr}$, et al. ACC/AHA Guidelines for Implantation of Cardiac Pacemakers and Antiarrhythmia Devices: Executive Summary. Circulation. 1998; 97(13): 1325-35.

7. Cox SM, Werner CL, Gilstrap LC, Cunningham FG, Hoffman B. Williams Obstetrics. 22nd ed. New York: McGraw-Hill Companies; 2005.

8. Michaëlsson M, Engle MA. Congenital heartblock: An international study of natural history. Cardiovasc Clin 1972; 4(3): 85-101.

9. Dalvi BV, Chaudhuri A, Kulkarni HL, Kale PA. Therapeutic guidelines for congenital complete heart block presenting in pregnancy. Obstet Gynecol 1992; 79(5): 802-804.

10. Kharde VV, Patil VV, Dhulkhand VK, Divekar DS. A Parturient with Complete Heart Block for Cesarean Section. J Anaesth Clin Pharmacol 2010; 26: 401-402.

11. Sullivan JM, Ramanathan KB. Management of medical problems in pregnancy - severe cardiac disease. N Engl J Med 1985; 313(5): 304309.

12. Hidaka N, Chiba $Y$, Kurita $T$, Satoh S, Nakano H. Is intrapartum temporary pacing required for women with complete atrioventricular block? An analysis of seven cases. BJOG. 2006; 113(5): 605-607.

13. Mehta S, Goswami D, Tempe A. Successful pregnancyoutcome in a patient with complete heart block. J Postgrad Med. 2003; 49(1): 98.

14. Güdal M, Kervancioğlu C, Oral D, Gürel T, Erol C, Sonel A. Permanent pacemaker implantation in apregnant woman with the guidance of ECG and two dimensional echocardiography. Pacing Clin Electrophysiol. 1987; $10(3):$ 543-545.

15. Lau CP1, Lee CP, Wong CK, Cheng CH, Leung WH. Rate responsive pacing with a minute ventilation sensing pacemaker during pregnancy and delivery. Pacing Clin Electrophysiol. 1990; 13(2): 158-163.

16. Modi MP, Butala B, Shah VR. Anaesthetic management of an unusual case of complete heart block for LSCS. Indian J Anaesth. 2006; 50(1): 43-44.

17. Suri V, Keepanasseril A, Aggarwal N, Vijayvergiya R, Chopra S, Rohilla M. Maternal complete heart block in pregnancy : Analysis of four cases and review management. J Obstet Gynaecol Res. 2009: 35(3): 434437.

18. Minassian VA1, Jazayeri A. Favorable Outcome in a Pregnancy With Complete Fetal Heart Block and Severe Bradycardia. Obstet Gynecol. 2002; 100 (5): 1087-9. 